\title{
Are Heart Muscle Irisin Levels and FNDC5 Gene Expression Regulated by Endurance and Resistance Exercises?
}

\author{
${ }^{1}$ Mohammad Hosseinzadeh, ${ }^{2}$ Amir Rashid Lamir ${ }^{*}$, ${ }^{3}$ Seyed Mahmud Hejazi \\ ${ }^{1}$ Department of Physical Education, Neyshabur Branch, Islamic Azad University, Neyshabur, Iran. ${ }^{2}$ Department of \\ Exercise Physiology, Faculty of Sport Science, Ferdowsi University of Mashhad, Mashhad, Iran. ${ }^{3}$ Department of \\ Physical Education, Mashhad Branch, Islamic Azad University, Mashhad, Iran.
}

\begin{abstract}
Background. Irisin is the result of the proteolysis of fibronectin type III domain-containing protein 5, the levels of which are determined by physical activity. Objective(s). This study aims to identify changes in FNDC5 gene expression and the heart muscle irisin levels in male rats following 8 weeks resistance and endurance trainings. Methods. Fifteen male rats (10-12 weeks old and 331.8 $\pm 63.09 \mathrm{~g}$ in weight) were randomly assigned to three groups: control, resistance and endurance training groups (5 in each group). The endurance group performed activities on a treadmill for 8 weeks. The resistance group performed activities for 8 weeks on a one-meter-long ladder with a slope of $85 \circ$. The control group did not undergo any particular training. Sampling was carried out $72 \mathrm{~h}$ after the end of the training. The heart tissue were excised, cleaned and immediately frozen in liquid nitrogen, and stored at $-70 \circ \mathrm{C}$. FNDC5 gene expression and the heart muscle irisin levels were measured using Real-Time PCR and ELISA, respectively. Results. Kruskal-Wallis test showed a significant increase in the heart muscle FNDC5 expression in both training groups $(\mathrm{P}=0.004)$. One-way ANOVA showed a significant difference in heart muscle irisin levels among the groups $(\mathrm{p}=0.004)$. Conclusion. It can be concluded that both resistance and endurance training can significantly increase FNDC5 gene expression and the heart muscle irisin levels, suggesting that physical activity can prevent and cure cardiovascular diseases.
\end{abstract}

\section{KEY WORDS: Resistance Training, Endurance Training, FNDC5, Irisin.}

\section{INTRODUCTION}

Cardiovascular diseases are the main cause of death worldwide. Diabetes, dyslipidemia, high blood pressure, family background, obesity and smoking can also play a role in enhancing the risk of cardiovascular diseases. Moreover, genetic factors can predispose individuals to the diseases. Recent studies have demonstrated the role of genetic and epigenetic factors in the pathogenesis of coronary diseases. However, the exact mechanism is not clearly known (1).

Among the largest muscles in the human body, is the heart muscle (myocardia), which releases several myokines. One of the myokines which is mainly expressed in the myocardium is irisin (2).

Irisin is a small polypeptide hormone, containing 111 amino-acids with a molecular

*. Corresponding Author:

Amir Rashid Lamir

E-mail: rashidlamir@um.ac.ir 
weight of $22 \mathrm{kDa}$ (3), which is the product of the proteolysis of a trans-membrane protein called fibronectin type III domain-containing protein 5 (FNDC5) (4), and also known as exercise hormone (5). FNDC5 has 209 amino-acid remnants (6) and its expression takes place in a number of organs, including the heart. The role it plays in the browning process of white adipose tissue has recently attracted the attention of several researchers (6-8).

The major function of irisin in white adipose cells is increasing the expression on uncoupled protein I (UCP1), which results in metabolic advantages by burning energy resources (9).

In this regard, Boström et al. (2012) showed that peroxisome proliferator-activated receptorgamma (PPAR- $\gamma$ ) coactivator-1 $\alpha$ (PGC1- $\alpha$ ) regulates FNDC5 gene expression and is responsible for the browning of white adipose tissue (10). As a result, the positive effects of PGC1 cause UCP-1 expression and thermogenesis in the brown adipose tissue (11).

Evidently, brown adipose tissue functions as a protector for the spine or backbone, as well as mediastinum, particularly in para-auortic zone, and also around the heart, especially the apex (12). Brown adipose is of low energizing potential and high thermogenesis (13). It has been observed that the browning of white adipose tissue can help protect against metabolic disorders caused by diet, obesity and metabolic diseases such as type II diabetes $(3,14)$. Because such disorders are closely related to cardiovascular diseases including coronary diseases (15), it is plausible that stimulating particular brown adipose sources or facilitating the alteration of white to brown adipose can be a promising strategy in treating metabolic disorders and cardiovascular diseases (16).

Taking extra calories and the reduction of physical activity play a role in development of such chronic diseases such as cardiovascular diseases (17).

Conversely, regular physical exercise is proportionate to atherosclerosis and the occurrence of primary and secondary cardiovascular attacks, although the mechanisms of physical exercise leading to prevention of atherosclerosis are not clearly known yet (18). Nonetheless, these are perhaps, a combination of optimal energy balance, fatness limitation and inflation, which is the potential result of the secretion of myokines (5).

In this regard, irisin is a myokine which is involved in the development of atherosclerosis (1) and thus can help in the treatment of atherosclerosis, as it is a potential treatment goal (18).

Its role in physiology is, however, quite unknown (15). Different studies on humans and animals have shown that irisin/FNDC5 expression is related to the intensity, duration and type of physical activity $(19,20)$.

For instance, Norhim et al. (2014) found an increase in irisin level immediately after the final session of combined (resistance and endurance) physical activities both in healthy and diabetic subjects (21). However, Kurdiova et al. (2014) failed to find any change in the levels of this peptide in obese and overweight subjects neither immediately nor $60 \mathrm{~min}$ after the last session of combined (resistance and endurance) physical activities (22).

The overall conclusion of these studies is that exercise is among the basic factors that stimulate the secretion of this hormone and its functioning upon energy metabolism (20).

On the other hand, the excitation parameter and the nature of the physical activity (as endurance, resistance or speed) is a decisive factor in this regard (20).

Whereas, metabolic adaptations due to aerobic exercise have often been the focus of attention, and the role of endurance training in the development of physical health and preparation - even in people with diverse physical conditions, is quite realized (23).

It has been also suggested that endurance training may be beneficial in energy expenditure and fat oxidation (24). Taking into consideration the metabolic properties of the brown adipose tissue as a working factor in the prevention and treatment of obesity, metabolic disorders and cardiovascular diseases (14), and the role of physical activity in the browning of white adipose tissue (25), a large number of researches is needed to determine, if physical activity can play a key role in the overall metabolism (26), the kind of activity and intensity, and duration needed to affect the factors involved in the browning of white adipose tissue. Thus, the current survey revealed that most studies had 
investigated serum irisin levels in unhealthy, elderly or obese individuals $(27,28)$ and that in the field of physical training, the results on FNDC5 gene expression and the irisin levels in the heart muscle as a tissue with a high potential of metabolism which contains both types of brown and white obesity tissues (12), producing a larger amount of irisin in comparison with the skeletal muscle (29), has attracted less attention. Therefore, the present study aims to investigate the expression of FNDC5 and irisin in the heart muscle following eight weeks of resistance and endurance trainings in male rats.

\section{MATERIALS AND METHODS}

With the aim of this experimental study, 15 male rats (Wistar rats, 10-12 weeks old with an average weight of $331.8 \pm 63.09 \mathrm{~g}$ ) were randomly assigned to three groups: control , resistance and endurance groups. These rats were kept in controlled light conditions (12 h of dark after $12 \mathrm{~h}$ of light conditions), temperature $\left(22 \pm 1^{\circ} \mathrm{C}\right)$ and humidity $(50 \%)$ in transparent polycarbonate cages for rodents and they had free access to food and water.

Exercise Training Protocol. Prior to the beginning of the training protocol, the subjects spent a week to familiarize with the procedure. The familiarization process for the aerobic group included 5 sessions of walking on the treadmill at a speed of $15-20 \mathrm{~m}$ per minute and a zero slope that lasted for $20-40 \mathrm{~min}$. For the resistance group, it consisted of 5 sessions of climbing a one-meter-high ladder with a slope of $60-80^{\circ}$. This followed attaching the clipper and the connected weight to the rat's tail. After wards, the endurance group performed the aerobic exercise, which was composed of 8 weeks (5 days a week) running on the treadmill at a speed of $20 \mathrm{~m} / \mathrm{min}$ for $60 \mathrm{~min}$ in each session. The first 10 min was allocated to warmup and the final $10 \mathrm{~min}$ was allocated to cooldown at a speed of $15 \mathrm{~m} / \mathrm{min}$ (30).

The resistance training was done with 3 sessions per week for 8 weeks. During the first week, the weight was connected to each subject's tail which is equal to $50 \%$ of the rat's body weight with a $10 \%$ weekly increase. The training was done on a one-meter-high ladder such that a weight was attached to the clipper on the rat's tail, and the animal had to climb up the ladder. This was done for three rounds at a repetition of five. The rounds' intervals were 3 min and the interval for repetitions was $1 \mathrm{~min}$. This training method was adapted from reliable sources $(31,32)$. The control group did no exercise over this period.

Tissue Biopsies. $72 \mathrm{~h}$ after the final exercise session, the rats were anaesthetized in the specialized sampling site (sterile space) with a combination of ketamine (30-50 mg for each $\mathrm{kg}$ of weight) and xylazine (3-5 mg for each $\mathrm{kg}$ of weight) (32). The heart was excised, cleaned, washed in ice cold saline, and immediately frozen in liquid nitrogen and stored at $-80^{\circ} \mathrm{C}$ until RNA extraction. Measurement of heart tissue irisin levels was done via ELISA method, using Rat irisin ELISA Kit (EK-067-52), Phoenix, USA.

RNA, cDNA Synthesis and Real-time PCR. So that mRNA could be taken, $50 \mathrm{mg}$ of the frozen tissue was taken for homogenization. In accordance with the developer's advice, Sinagen RNA-Plus kit was applied in order to remove mRNA. Two micrograms of mRNA was taken from each sample to synthesize the complementary DNA (cDNA) (cDNA Synthesis Kir, BIONEER, Korea). Table 1 shows the nucleotide primer sequences of FNDC5 (32) and the reference genes (33).

Table 1.Oligonucleotide primer sequences

\begin{tabular}{ccr}
\hline Gene & Type & primer sequences \\
\hline \multirow{2}{*}{ FNDC5 } & forward & 5-GTCTCCCACCACCATCTT-3 \\
& Reverse & 5-TCTGTCTCTGAGTGTAGCCTTAGC-3 \\
\hline \multirow{2}{*}{ GAP.DH } & forward & 5'-GTGCCAGCCTCGTCTCATAG \\
& Reverse & 5'-GACTGTGCCGTTGAACTTGC \\
\hline
\end{tabular}

Real Time PCR was done to measure the level of gene expression and the parameters for a two-step PCR were $95^{\circ} \mathrm{C}$ for $5 \mathrm{~min}, 1$ cycle, then $95^{\circ} \mathrm{C}$ for $10 \mathrm{~s}$ and then $60^{\circ} \mathrm{C}$, for $30 \mathrm{~s}, 45$ cycles, 
and parameters for melting curve program were 55 to 95 ( 0.5 increment for $\mathrm{f}$ seconds).

After the reaction, the threshold cycle $(\mathrm{Ct})$ was calculated for each sample. The proportion of the gene $\mathrm{Ct}$ to the housekeeping gene that produced the level of gene expression was determined using applying Livak equation (34).

Statistical Analysis. Wilk test was done and there was no normal distribution of data with regards to heart tissue FNDC5 gene expression. Then, for comparison of the mean, statistical non-parametric Kruskal-Wallis test was done, and Mann Whittney test was applied to settle the inter-group significance. Moreover, due to the normal distribution of heart irisin levels, oneway ANOVA and Tukey test were done. The level of significance was set at $\mathrm{P}>0.05$. SPSS 16 was used to analyze the data.

\section{RESULTS}

Changes in heart muscle FNDC5 gene expression. Results of Shapiro-Wilk test showed no normal distribution of data related to FNDC5 gene expression and a normal distribution of the heart muscle irisin levels. Kruskal-Wallis test showed a significant difference in FNDC5 gene expression among the groups $(\mathrm{P}=0.004$, Chi-Square $=10.909)$. Mann Whitney test was used to analyze intergroup differences and the results showed that there was a significant difference in FNDC5 expression between the resistance and endurance groups on one hand and the control group on the other hand $(\mathrm{P}=0.005 \& \mathrm{P}=0.005$, respectively). However, no significant difference in FNDC5 gene expression was found between the aerobic training and endurance groups $(\mathrm{P}=0.11)$ (Figure 1).

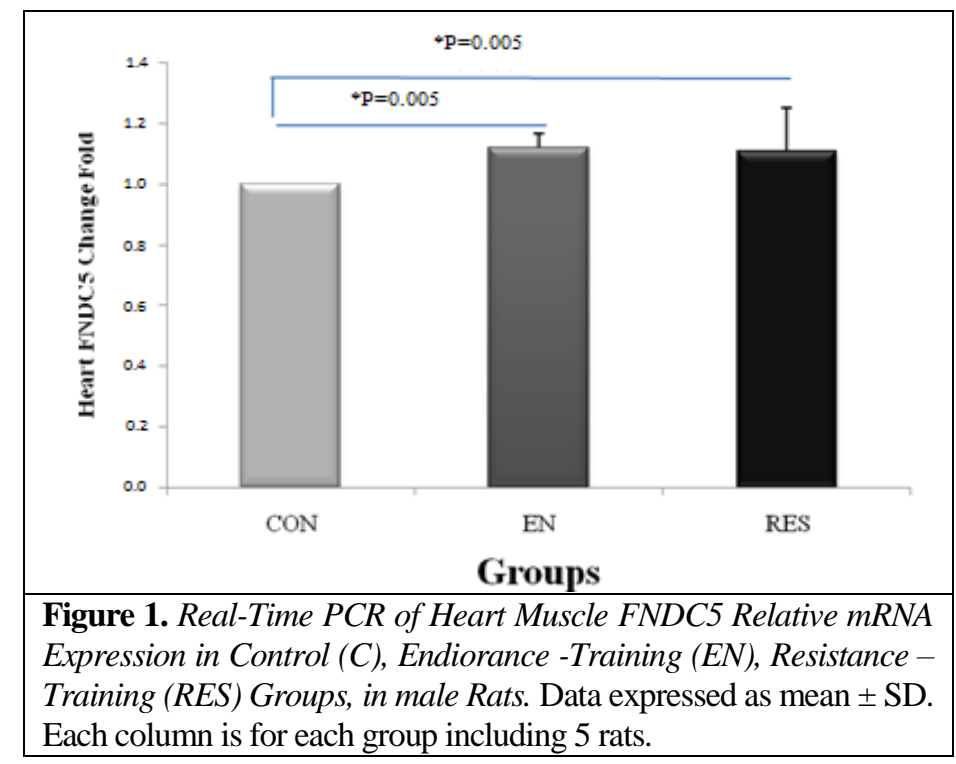

Changes in heart muscle irisin levels. The heart muscle irisin levels were normally distributed and one-way ANOVA showed a significant difference among the groups $(\mathrm{P}=$ $0.001 \& \mathrm{~F}=53.91)$. Tukey test showed that there was a significant increase in the heart muscle irisin levels in the resistance and endurance groups as compared to the control group ( $\mathrm{P}=$ 0.001 and $\mathrm{P}=0.001$, respectively). It also showed a significant difference between the training groups $(\mathrm{P}=0.037)$ (Figure 2).

\section{DISCUSSION}

The findings of the present study showed that 8 weeks' resistance and endurance activities caused a significant increase in FNDC5 gene expression and irisin levels in the heart tissue.

The heart tissue is known as an irisinsecreting organ (35). In vivo and in vitro studies indicate the major role that irisin plays in the functioning of the heart muscle (36) and is a promising candidate in treating metabolic disorders and cardiovascular diseases. This 
hormone is the protolithic product of FNDC5, whose expression is stimulated by physical activities and positive regulation of PPAR- $\gamma$ and PGC1- $\alpha$ (35). It was recently reported that irisin responded to resistance and endurance trainings.
Yet, there is incongruity among researchers as to what kind of training (endurance vs. resistance) with what intensity (high vs. medium) and what duration (acute vs. chronic) leads to more desirable results (37).

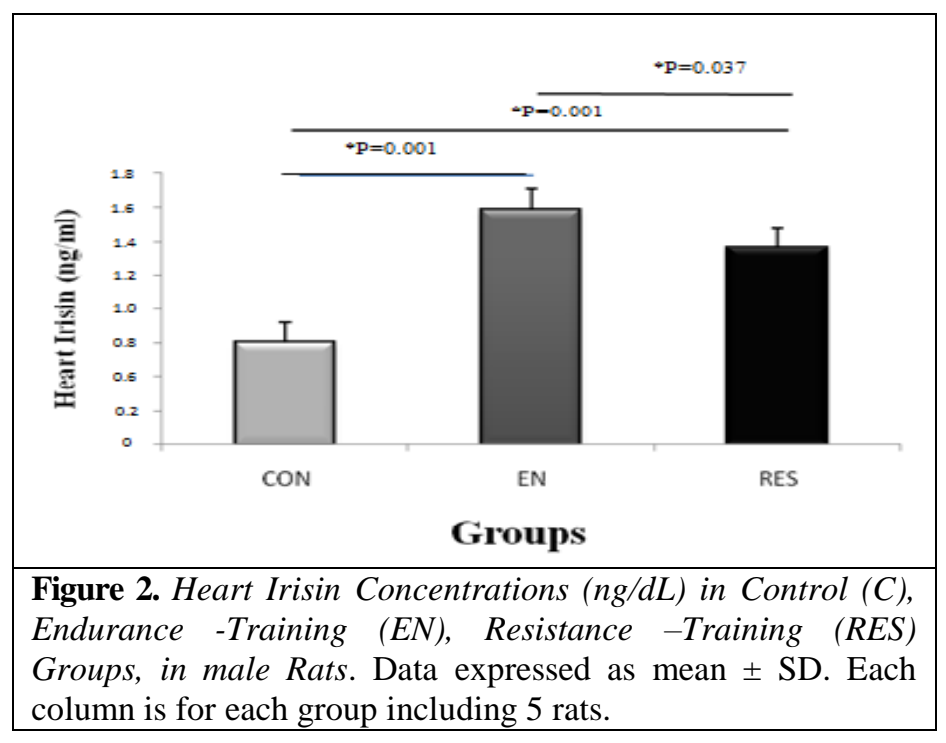

The present study showed that eight weeks' resistance and endurance training can almost equally increase FNDC5 gene expression and irisin levels, which is in accordance with previous researches $(21,32,35,37,38)$. For instance, Reisi et al. (2015) observed increase in male rats' fat tissue FNDC5 gene expression after 8 weeks' endurance training (32). Also, Aydin et al. (2014) observed a significant increase in the heart muscle irisin in rats (young or old) after exercise (floating on water) (15). However, these study findings contradict those of Fein et al. (2013), who reported that there was no significant difference in FNDC5 gene expression in epicardial or subcutaneous fat, deltoid muscle, triceps brachii muscle or heart muscle in pigs (39). The findings were also different from those of Pekkala et al. (2013), who reported no increase in serum FNDC5 and irisin following intense long-term aerobic exercises and a combination of aerobic and endurance trainings in young or old men. Just a single session of endurance training increased FNDC5 gene expression in young men (40). These diverse findings suggest that the different training protocols, particularly when applied to different species (humans vs. Guinea pigs), yield incomparable results (15).

The mechanisms that depict the protective effects of physical exercise are yet to be known. Endurance training is necessary for most metabolic adaptations, while resistance training is potentially valuable too. Endurance training can help improve health by augmenting fatless mass and fat oxidation (41). Research shows that both aerobic (resistance) and endurance (power) training reduce the profile of cardiovascular risk and increase basic metabolism (35). It seems that the irisin levels increase in male rats in adaptation to endurance training, which can then make white subcutaneous adipose tissue to get brown and increase energy expenditure via thermogenesis that is not induced by physical activity nor by food consumption (41). Another favorable effect of endurance training is increasing fatless mass, which can give rise to resting metabolism and energy expenditure (41). Research supports the existence of irisin receptors on the surface of heart cells, through which irisin can activate several lower-grade signaling routes in cardio myoblasts (35). It has been suggested that irisin might induce its effects (growth, thermogenesis, etc.) on heart cells by 
regulating the course of $\mathrm{P} 13 \mathrm{~K}-\mathrm{AKT}$; that is, these courses play their part in the browning of white adipose cells in rats via irisin (35).

Another mechanism that could help explain the effect of physical activity on heart functioning through increasing irisin might be related to excessive FNDC5 expression following physical activities, which in turn could increase the expression of PPARs in the white adipose tissue. In other words, PPAR may have a mediating role in FNDC5 effects (36). A large number of PPAR-a target genes are involved in the removal of cellular fatty acids (like lipase lipoprotein) and in the beta-oxidation of mitochondrial fatty acids. In congruence with this function, PPAR-a is highly expressed in brown adipose tissue, which is actually a characteristic of brown adipose tissue, as contrasted with white adipose tissue, and together with PGC-1a, regulates the principal parts of thermogenesis like UCP-1. That is why PGC-1a is known as an immediate target of regulating the coupling of PPAR-a in brown adipose tissue (16). Research has suggested PPAR gene expression after resistance training (42-44). Therefore, one of the ways through which resistance training affects irisin levels might be via increase in PPAR gene expression, which in turn, positively regulates PGC-1a and UCP-1.

On the other hand, it has been suggested that irisin peptides produced in the heart muscle can directly prevent the formation of plaques (athroms) by browning white adipose tissue. Thus, metabolism is not paired and the heart burns more calories (15). Therefore, one of the mechanisms through which the heart benefits from physical activities through the increase of irisin levels might be the major role irisin plays in maintaining the functioning of endothelial cells. Irisin has thus been known as a potential medicinal aid in treating atherosclerosis (18).

\section{CONCLUSION}

The present research showed that both resistance and endurance trainings increase the expression of FNDC5 and the amount of irisin in the heart tissue. It is probably through this way that they improve cardiovascular conditions. Considering the limitations of the present study in measuring white adipose tissue and how much browning took place, it is suggested that future research should pay attention on these factors so that the role of physical activity in increasing irisin levels and the mechanisms at work in the browning of white adipose tissue are better understood.

\section{APPLICABLE REMARKS}

- Irisin is a peptide which is synthesized in the heart muscle and can directly inhibit the formation of atheroma by stimulating the conversion of white adipose tissue into brown adipose tissue, thus increasing metabolic uncoupling and hence caloric expenditure in the heart.

\section{REFERENCES}

1. Deng W. Association of Serum Irisin Concentrations with Presence and Severity of Coronary Artery Disease. Medical Science Monitor. 2016;22:4193-7.

2. Anastasilakis AD, Koulaxis D, Kefala N, Polyzos SA, Upadhyay J, Pagkalidou E, et al. Circulating irisin levels are lower in patients with either stable coronary artery disease (CAD) or myocardial infarction (MI) versus healthy controls, whereas follistatin and activin A levels are higher and can discriminate MI from CAD with similar to CK-MB accuracy. Metabolism. 2017;73:1-8.

3. Zhang Y, Li R, Meng Y, Li S, Donelan W, Zhao Y, et al. Irisin stimulates browning of white adipocytes through mitogen-activated protein kinase p38 MAP kinase and ERK MAP kinase signaling. Diabetes. 2014;63(2):514-25.

4. Ost M, Coleman V, Kasch J, Klaus S. Regulation of myokine expression: Role of exercise and cellular stress. Free radical biology \& medicine. 2016;98:78-89.

5. Rabiee F, Forouzanfar M, Ghazvini Zadegan F, Tanhaei S, Ghaedi K, Motovali Bashi M, et al. Induced expression of Fndc5 significantly increased cardiomyocyte differentiation rate of mouse embryonic stem cells. Gene. 2014;551(2):127-37.

6. Aydin S. Three new players in energy regulation: preptin, adropin and irisin. Peptides. 2014;56:94-110. 
7. Barja-Fernandez S, Folgueira C, Castelao C, Al-Massadi O, Bravo SB, Garcia-Caballero T, et al. FNDC5 is produced in the stomach and associated to body composition. Scientific reports. 2016;6:23067.

8. Schumacher MA, Chinnam N, Ohashi T, Shah RS, Erickson HP. The structure of irisin reveals a novel intersubunit beta-sheet fibronectin type III (FNIII) dimer: implications for receptor activation. The Journal of biological chemistry. 2013;288(47):33738-44.

9. Bonfante IL, Chacon-Mikahil MP, Brunelli DT, Gaspari AF, Duft RG, Lopes WA, et al. Combined training, FNDC5/irisin levels and metabolic markers in obese men: A randomised controlled trial. European journal of sport science. 2017;17(5):629-37.

10. Bostrom P, Wu J, Jedrychowski MP, Korde A, Ye L, Lo JC, et al. A PGC1-alpha-dependent myokine that drives brown-fat-like development of white fat and thermogenesis. Nature. 2012;481(7382):463-8.

11. Wenza T, Rossib SG, Rotundob RL, Spiegelmanc BM, Moraesa CT. Increased muscle PGC-1a expression protects from sarcopenia and metabolic disease during aging. Proc Natl Acad Sci U S A. 2009;111(44):15851.

12. Nedergaard J, Bengtsson T, Cannon B. Unexpected evidence for active brown adipose tissue in adult humans. American journal of physiology Endocrinology and metabolism. 2007;293(2):E444-52.

13. Kajimura S, Saito M. A new era in brown adipose tissue biology: molecular control of brown fat development and energy homeostasis. Annual review of physiology. 2014;76:225-49.

14. Raschke S, Elsen M, Gassenhuber H, Sommerfeld M, Schwahn U, Brockmann B, et al. Evidence against a beneficial effect of irisin in humans. PloS one. 2013;8(9):e73680.

15. Aydin S, Kuloglu T, Aydin S, Eren MN, Celik A, Yilmaz M, et al. Cardiac, skeletal muscle and serum irisin responses to with or without water exercise in young and old male rats: cardiac muscle produces more irisin than skeletal muscle. Peptides. 2014;52:68-73.

16. Hondares E, Rosell M, Diaz-Delfin J, Olmos Y, Monsalve M, Iglesias R, et al. Peroxisome proliferator-activated receptor alpha (PPARalpha) induces PPARgamma coactivator 1alpha (PGC-1alpha) gene expression and contributes to thermogenic activation of brown fat: involvement of PRDM16. The Journal of biological chemistry. 2011;286(50):43112-22.

17.Li S, Laher I. Exercise Pills: At the Starting Line. Trends in pharmacological sciences. 2015;36(12):906-17.

18.Zhang Y, Mu Q, Zhou Z, Song H, Zhang Y, Wu F, et al. Protective Effect of Irisin on Atherosclerosis via Suppressing Oxidized Low Density Lipoprotein Induced Vascular Inflammation and Endothelial Dysfunction. PloS one. 2016;11(6):e0158038.

19. Khodadadi H, Rajabi H, Attarzadeh R, Abbasian S. The Effect of High Intensity Interval Training (HIIT) and Pilates on Levels of Irisin and Insulin Resistance in Overweight Women. Iranian Journal of Endocrinology and Metabolism. 2017;16(3):190-6.

20. Jones AM, Carter H. The Effect of Endurance Training on Parameters of Aerobic Fitness. Sports Med. 2000;29 (6):373-86.

21. Norheim F, Langleite TM, Hjorth M, Holen T, Kielland A, Stadheim HK, et al. The effects of acute and chronic exercise on PGC-1alpha, irisin and browning of subcutaneous adipose tissue in humans. The FEBS journal. 2014;281(3):739-49.

22. Kurdiova T, Balaz M, Vician M, Maderova D, Vlcek M, Valkovic L, et al. Effects of obesity, diabetes and exercise on Fndc5 gene expression and irisin release in human skeletal muscle and adipose tissue: in vivo and in vitro studies. The Journal of physiology. 2014;592(5):1091-107.

23. Reisi J, Rajabi H, ghaedi K, Marandi SM, Dehkhoda MR. Effect of Acute Resistance Training on Plasma Irisin Protein Level and Expression of Muscle FNDC5 and Adipose Tissue UCP1 Genes in Male Rats. Journal of Isfahan Medical School. 2013;31(256).

24.Hunter GR, Wetzstein CJ, Fields DA, Brown A, Bamman MM. Resistance training increases total energy expenditure and free-living physical activity in older adults. J Appl Physiol (1985). 2000;89(3):977-84.

25. Stanford KI, Middelbeek RJ, Goodyear LJ. Exercise Effects on White Adipose Tissue: Beiging and Metabolic Adaptations. Diabetes. 2015;64(7):2361-8.

26. Sanchez-Delgado G, Martinez-Tellez B, Olza J, Aguilera CM, Gil A, Ruiz JR. Role of Exercise in the Activation of Brown Adipose Tissue. Annals of nutrition \& metabolism. 2015;67(1):21-32.

27. Icli A, Cure E, Cumhur Cure M, Uslu AU, Balta S, Arslan S, et al. Novel myokine: irisin may be an independent predictor for subclinic atherosclerosis in Behcet's disease. Journal of investigative medicine : the official publication of the American Federation for Clinical Research. 2016;64(4):875-81.

28. Lu J, Xiang G, Liu M, Mei W, Xiang L, Dong J. Irisin protects against endothelial injury and ameliorates atherosclerosis in apolipoprotein E-Null diabetic mice. Atherosclerosis. 2015;243(2):438-48.

29. Efe TH, Acar B, Ertem AG, Yayla KG, Algul E, Yayla C, et al. Serum Irisin Level Can Predict the Severity of Coronary Artery Disease in Patients with Stable Angina. Korean circulation journal. 2017;47(1):44-9. 
30. Disanzo BL, You T. Effects of exercise training on indicators of adipose tissue angiogenesis and hypoxia in obese rats. Metabolism. 2014;63(4):452-5.

31. Matinhomaee H ZS, Azarbayjani M and Piri M. Effects of Boldenone consumption and resistance exercise on hepatocyte morphologic damages in male wistar rats. Pelagia Research Library. 2014:211-4.

32. Reisi J, Rajabi H, Ghaedi K, Marandi SM, Asadisamani Z, Kazeminasab F. Effect of eight weeks' resistance training on plasma irisin protein level and muscle FNDC5 and adipose tissue UCP1 genes expression in male rats. sport physiology. 2015;28:117-30.

33. Ghanbari-Niaki A, Ghanbari-Abarghooi S, Rahbarizadeh F, Zare-Kookandeh N, Gholizadeh M, Roudbari F, et al. Heart ABCA1 and PPAR- alpha Genes Expression Responses in Male rats: Effects of High Intensity Treadmill Running Training and Aqueous Extraction of Black Crataegus-Pentaegyna. Res Cardiovasc Med. 2013;2(4):1539.

34. Livak KJ, Schmittgen TD. Analysis of relative gene expression data using real-time quantitative PCR and the 2(Delta Delta C(T)) Method. Methods. 2001;25(4):402-8.

35. Xie C, Zhang Y, Tran TD, Wang H, Li S, George EV, et al. Irisin Controls Growth, Intracellular Ca2+ Signals, and Mitochondrial Thermogenesis in Cardiomyoblasts. PloS one. 2015;10(8):e0136816.

36. Esfahani M, Baranchi M, Goodarzi MT. Irisin and Metabolic Disorders. Avicenna J Med Biochem. 2016;4(1).

37. Kim HJ, So B, Choi M, Kang D, Song W. Resistance exercise training increases the expression of irisin concomitant with improvement of muscle function in aging mice and humans. Experimental gerontology. 2015;70:11-7.

38. Lecker SH, Zavin A, Cao P, Arena R, Allsup K, Daniels KM, et al. Expression of the irisin precursor FNDC5 in skeletal muscle correlates with aerobic exercise performance in patients with heart failure. Circulation Heart failure. 2012;5(6):812-8.

39. Fain JN, Company JM, Booth FW, Laughlin MH, Padilla J, Jenkins NT, et al. Exercise training does not increase muscle FNDC5 protein or mRNA expression in pigs. Metabolism. 2013;62(10):1503-11.

40. Pekkala S, Wiklund PK, Hulmi JJ, Ahtiainen JP, Horttanainen M, Pollanen E, et al. Are skeletal muscle FNDC5 gene expression and irisin release regulated by exercise and related to health? The Journal of physiology. 2013;591(21):5393-400.

41. Reisi J, Ghaedi K, Rajabi H, Marandi SM. Can Resistance Exercise Alter Irisin Levels and Expression Profiles of FNDC5 and UCP1 in Rats? Asian journal of sports medicine. 2016;7(4):e35205.

42. Butcher LR, Thomas A, Backx K, Roberts A, Webb R, Morris K. Low-intensity exercise exerts beneficial effects on plasma lipids via PPARgamma. Medicine and science in sports and exercise. 2008;40(7):1263-70.

43. Russell AP, Feilchenfeldt J, Schreiber S, Praz M, Crettenand A, Gobelet C, et al. Endurance training in humans leads to fiber type-specific increases in levels of peroxisome proliferator-activated receptor-gamma coactivator-1 and peroxisome proliferator-activated receptor-alpha in skeletal muscle. Diabetes. 2003;52(12):2874-81.

44. Petridou A, Tsalouhidou S, Tsalis G, Schulz T, Michna H, Mougios V. Long-term exercise increases the DNA binding activity of peroxisome proliferator-activated receptor gamma in rat adipose tissue. Metabolism. 2007;56(8):1029-36. 